\title{
An Overview of Mixed and Multi Method Research
}

\author{
Faiswal Kasirye \\ Department of Communication, \\ International islamic University Malaysia \\ Email: Kasirye.faiswal@gmail.com
}

\section{INTRODUCTION}

Merging quantitative and qualitative research methods is currently a common practice especially in social sciences research. These two approaches have corresponding strengths and that leveraging on them improves research strengths in finding research relationships. Weller and Barnes (2017) argue that "there are two distinct approaches that combine quantitative and qualitative work called multi-method and mixed-method research" (p. 1020). Multi-method research rests on triangulation that seeks the merging of results under the different methods. Similarly, mixed-method research rests on the possible outcomes approach to underlying interpretation. It is incredulous that case studies can achieve causal interpretation but instead, the method seeks to create a division of labor among methods where large works provide evidence of the underlying relationship while case studies provide critical perceptions into the validity of the assumptions of the studies, their scope, and the hypotheses of the study (Weller \& Barnes, 2017).

Research as a phenomenon has been known for qualitative and quantitative approaches however, depending on which approach you prefer, there have emerged some other approaches that have been able to combine the two paradigms and placed them into mixed and multi methods. Some studies have so far been able to recognize mixed methods as a qualitative method while others make it distinct on its own. There are however other researchers who have placed mixed method on both qualitative and quantitative approaches in form of method in just a single study, never the less, mixed methods can also be exclusively quantitative while others qualitative (Kroll \& Neri 2009).

For multimethod, it has also emerged as a possible solution to the very many glaring issues in the scientific research and answering as any questions and possible (Greene 2015). Multimethod started with getting results to understand people's behaviour in their social lives as opposed to 
mixed methods that came with making a difference in research methods through mixing different data at the various levels of research. Multimethod started in the field of anthropology and program evaluation (Denzin 1978 and Cook 1985). This write up distinguishes between the two research methods as follows;

\section{Mixed Method Research Approach}

Mixed method research is currently seen as one of the main research approaches that define the modern way of doing research (Giddings \& Grant, 2007 and Johnson et al. 2007). Creswell (2007) crafted a leading definition to the mixed method approach and referred to it as "research in which the investigator collects and analyses data, integrates the findings, and draws conclusions using both qualitative and quantitative approaches or methods in a single study or a program of inquiry" (p. 4). In addition, understanding the importance and advantages of both qualitative and quantitative methods while doing mixed methods is very paramount because everything done is in relation to the two methods (Anguera et al., 2018).

Creswell (2015) notes that the approach has been developing for many years especially in the different social sciences fields. In addition, apart from qualitative and quantitative as paradigms, mixed methods is also recognized as the third approach that literally combines the former two approaches into developing a more vivid research although emerging with some methodological problems (Johnson et al., 2007).

Teddie and Tashakkori (2010) also went ahead to define mixed methods as "research designs using qualitative and quantitative data collection and analysis techniques in either parallel or sequential phases" (p. 11). The main issue in mixed method is to provide a more detailed formula and guidance in extracting data from the respondents in order to make replicable and generalizable findings right from the onset of the research process.

The mixed method approach however also comes with shortcomings that hinder the process although several researchers have tried to unpack the disadvantages of combining qualitative and quantitative methods into a one robust approach thereby referred to as the mixed method research paradigm (Johnson, 2012). 


\section{Multimethod Research Approach}

For multimethod, there have been several studies that have tried to explain the approach with a view of answering complex research questions I social sciences research (Greene 2015). Researchers in this approach may choose different methodological analyses of the same types or approach. This can be either using more than one qualitative research methods or choosing two different quantitative methods. The usage of such methodological approaches is referred to as multi-method research (Creswell, 2007). It should be noted that using two different methods from two different paradigms will automatically fall under mixed methods rather than that that uses two or more research methods from one paradigm at a time. Therefore, multimethod works with choosing from one paradigm or approach but choosing to use different methods to analyze your research project (Johnson \& Onwuegbuzie, 2004). Multi methods also increases the chances of getting varied and extensive research findings.

The other way to answer the question of multimethod is simply to combine a list of all research methodologies from one approach and then conduct a research using two or more methods from the same approach (Creswell \& Plano Clark, 2011).

In addition, Tashakkori and Teddlie (2003) define multiple method as "research in which more than one method or more than one worldview is used" (p. 11). Three categories are seen here which are; multimethod research, mixed method research, and mixed model research. It can be concluded that the distinction among these terms is related to the research stage of the study through "definition, of research questions, research methods, data collection and analysis, and the inference process"

Therefore, Morse (2003) also defines Multimethod design as the conduct of two or more research methods in one project. The results are then triangulated to form a complete whole. Similarly, the researcher differentiates multimethod and mixed method design in a way that multimethod design handles all projects in a complete way using the same methods from either qualitative or quantitative designs. In addition, Tashakkori and Teddlie (2003) also proposes the term mixed model research to represent the mixture of methods in many stages of any study. 


\section{Mixed vs Multi-Method Approaches}

There have been varying confusions relating to these two concepts of mixed and multi methods. However, their differences are more on the terminologies and the design as well as the methods they both use during their studies. Some researchers are disturbed by the way mixed methods are adopted by other researchers who have not yet gotten a clear distinction or boundary between mixed and multi method and conclusions have not yet been reached (Creswell, 2015).

In fact, some researchers still confuse mixed methods as multimethod and some of them contend that it's a combination of both quantitative and qualitative (Stange et al., 2006). To a surprise, some journals like the international journal of multiple research approaches failed to distinguish between the two new approaches by at least publishing a single article articulating the issues surrounding them, although by virtue of its name, you would think that they would come up with a clear approach to the questions relating to the two methods and publish it for the public as put forward by Burns et al. (2014).

Johnson et al. (2007) in trying to draw a distinction between the two said that, "Multimethod research is when different approaches or methods are used in parallel or sequence but are not integrated until inferences are being made" (p. 119). In all the literature sampled in this write up, most of them agree almost on the same aspects as Johnson does. Therefore, iam also inclined to support his definition because it resonates with me in regards to multimethods approach because it carries all types of research methods and I agree that they can all be used in one research study to come up with the different results or the same results.

Similarly, on mixed methods, it is defined as "a term used to combine qualitative and quantitative research methods in the same research project" (Johnson et al. 2007, p. 120). On that note, multimethod research may defer to indicate that different styles of research may be combined in the same research project thereby called a mixed method study. "These need not be restricted to quantitative and qualitative although, they may as well include qualitative participant observation with qualitative in-depth interviewing. Alternatively, it could include quantitative survey research with quantitative experimental research. And of course it would include quantitative with qualitative styles" (Johnson et al. 2007, p. 120). 
We can clearly say that the confusion that there is in the two concepts emanates from the conceptual definitions of the several researchers as reviewed above but also, and most importantly, it comes from the methodological approaches depending on the particular researcher's field of study. Most of them define the concepts from the study perspectives and fields.

A constant look at both approaches however brigs to a conclusion that integrating both designs into one study might be very instrumental in delivering rich data and findings, however, multimethod might not necessarily require one to do that because its study nature is a little bit different from that of the mixed methodology (Plano Clark and Ivankova, 2016). However, when the same goal and objective are at play, both qualitative and quantitative approaches may come in to achieve a similar goal and not when their aims and objectives are different.

Hunter and Brewer (2015a) contend that, "Multimethod research may be broadly defined as the practice of employing two or more different methods or styles of research within the same study or research program rather than confining the research to the use of a single method" (p. 187). This issue has already been reechoed by several researchers above however, mixed method is also along the same line although differs a bit on the route to using the different designs in one study or call it research methods in one research study. In a nutshell, multimethod studies are considered by the coexistence of different methodologies i.e. observational and selective, or observational and ethnographic (Anguera et al. 2014).

The distinction is that mixed methods combines qualitative and quantitative methods, while multimethods uses two qualitative methods where in principle, multi-methods research could also use two quantitative methods, the distinction is even clearer by Creswell's (2015) conclusions following his comment in reference to mixed methods: "A core assumption of this approach is that when an investigator combines statistical trends (quantitative data) with stories and personal experiences (qualitative data), this collective strength provides a better understanding of the research problem than either form of data alone', (p. 1).

Creswell (2015) goes on to explain what differentiates mixed methods from multimethods: "Mixed methods further is not simply the collection of multiple forms of qualitative data, nor the collection of multiple types of quantitative data. It involves the collection, analysis and integration of both quantitative and qualitative data. In this way, the value of the different approaches to research can contribute more to understanding a research problem that one form of data collection could on its 
own. When multiple forms of qualitative or quantitative data are collected, the term is 'multimethod' (Creswell 2015, pp. 2-3).

Lastly, Fetters and Molina-Azorin (2017) also contend in their issued statement on the issue of multi and mixed method that, "multi-methods research is a broader category that contains any two different methods, while mixed methods is a subset of that, where there are both qualitative and one quantitative methods." (p. 39).

In addition, Creswell (2011) concludes on this issue by saying, "Writers in mixed methods are also careful to distinguish 'multi-method studies' in which multiple types of qualitative or quantitative data are collected (see Creswell \& Plano Clark, 2007) from 'mixed methods studies' that incorporate collecting both qualitative and quantitative data." (p. 273).

\section{CONCLUSION}

In conclusion therefore, mixed method and multimethods are used interchangeably and differently by several researchers depending on their fields of study. this disagreement in the varied approaches to the two issues discussed above may continue to bring about confusion in the minds of junior and or young researchers who aspire to use any of the two methods. Therefore, there is an urgent need to straighten and strengthen the issue of mixed as well as multimethod research in order to help in guiding upcoming researchers and students who have interests in academic research.

To some researchers, multi-methods are similar or the same as mixed methods where by different design techniques i.e. data collection and data analysis within the same paradigm (quantitative or qualitative). Whereas for mixed methods, many refer to it as the third main research paradigm, where a single study that has a broader and logical approach that requires more than one research method to address it. These then apply quantitative and qualitative combined together in a single study, although there are many variations on that. 


\section{REFERENCES}

Anguera, M. T., Blanco-Villaseñor, A., Losada, J. L., Sánchez-Algarra, P., \& Onwuegbuzie, A. J. (2018). Revisiting the difference between mixed methods and multimethods: Is it all in the name? Quality \& Quantity, 52(6), 2757-2770.

Anguera, M. T., Camerino, O., Castañer, M., \& Sánchez-Algarra, P. (2014). Mixed methods en actividad física y deporte. Rev. Psicol. Dep, 23, 123-130.

Barnes, J., \& Weller, N. (2017). Case studies and analytic transparency in causal-oriented mixedmethods research. PS: Political Science \& Politics, 50(4), 1019-1022.

Burns, K., Bellows, M., Eigenseher, C., Jackson, K., Gallivan, J., \& Rees, J. (2014). Exploring patient engagement practices and resources within a health care system: applying a multiphased mixed methods knowledge mobilization approach. International Journal of Multiple Research Approaches, 8(2), 233-247.

Clark, V. P., \& Ivankova, N. V. (2016). How to use mixed methods research? Understanding the basic mixed methods designs. In Mixed methods research: A guide to the field (pp. 105134). SAGE Publications Ltd.

Cook, T. D. (1985). Post-positivist critical multiplism. In Reproduced in evaluation studies review annual. Sage Publications 2, 21-62, Beverly Hills.

Creswell, J. W. (2011). Controversies in mixed methods research. The Sage handbook of qualitative research, 4, 269-284.

Creswell, J. W. (2015). Revisiting mixed methods and advancing scientific practices. In The Oxford handbook of multimethod and mixed methods research inquiry.

Creswell, J. W., \& Plano Clark, V. L. (2011) Designing and Conducting Mixed Methods Research (2nd ed.). Thousand Oaks, CA: Sage Publications 3, 53-106.

Creswell, J. W., \& Tashakkori, A. (2007). Differing perspectives on mixed methods research.

Denzin, N. (1978). K. The research act. New York: McGraw-Hill Book Company, 2, 291-302.

Fetters, M. D., \& Molina-Azorin, J. F. (2017). The Journal of Mixed Methods Research starts a new decade: The mixed methods research integration trilogy and its dimensions.

Giddings, L. S., \& Grant, B. M. (2007). A Trojan horse for positivism? A critique of mixed methods research. Advances in nursing science, 30(1), 52-60.

Goertz, G., \& Mahoney, J. (2012). A tale of two cultures: Qualitative and quantitative research in the social sciences. Princeton University Press.

Greene, J. C. (2015). Preserving distinctions within the multimethod and mixed methods research merger. In The Oxford handbook of multimethod and mixed methods research inquiry. 5, 606-615. Oxford University Press, Oxford. 
Greene, Jennifer, Valerie Caracelli, and Wendy Graham (1989). 'Toward a Conceptual Framework for Mixed-Method Evaluation Designs.' Educational Evaluation and Policy Analysis 11(3), 255-274.

Hunter, A., \& Brewer, J. D. (2015). Designing multimethod research. In The Oxford handbook of multimethod and mixed methods research inquiry.

Johnson, R. B. (2012). Dialectical pluralism and mixed research. Am. Behav. Sci. 56(6), 751-754.

Johnson, R. B., \& Onwuegbuzie, A. J. (2004). Mixed methods research: A research paradigm whose time has come. Educational researcher, 33(7), 14-26.

Johnson, R. B., \& Onwuegbuzie, A. J. (2004). Mixed methods research: A research paradigm whose time has come. Educational researcher, 33(7), 14-26.

Johnson, R. B., Onwuegbuzie, A. J., \& Turner, L. A. (2007). Toward a definition of mixed methods research. Journal of mixed methods research, 1(2), 112-133.

Kroll T, Neri M 2009 Designs for mixed methods research. In: Andrew S, Halcomb E J (eds) Mixed Methods Research for Nursing and the Health Sciences. Wiley-Blackwell, Chichester, UK, pp 31-49.

Morse, J. M. (2003). Principles of mixed methods and multimethod research design. A. Tashakkori, \& C. Teddlie (Eds.), Handbook of mixed methods in social and behavioral research (pp. 189-208).

Šerá, E. (2017). Gary Goertz-James Mahoney: A Tale of Two Cultures. Qualitative and Quantitative Research in the Social Sciences. Historická sociologie, 9(2), 146-150.

Stange, K. C., Crabtree, B. F., \& Miller, W. L. (2006). Publishing multimethod research. Ann. Fam. Med. 4, 292-294.

Tashakkori, A., \& Teddlie, C. (2003). Handbook on mixed methods in the behavioral and social sciences. 1, 13-50.

Tashakkori, A., \& Teddlie, C. (2003). Issues and dilemmas in teaching research methods courses in social and behavioural sciences: US perspective. International Journal of Social Research Methodology, 6(1), 61-77.

Teddlie, C., \& Tashakkori, A. (2010). Overview of contemporary issues in mixed methods research. Handbook of mixed methods in social and behavioral research, 2, 1-41. 